\title{
Ureteral rupture after blunt abdominal trauma in a child with unknown horseshoe kidney
}

\author{
Arianna Mariotto, Nicola Zampieri, Mariangela Cecchetto, Francesco Saverio Camoglio \\ Pediatric Surgical Unit, Department of Surgical Sciences, G.B. Rossi Hospital, Verona, Italy
}

\begin{abstract}
More than $90 \%$ of renal injuries in children result from blunt abdominal trauma. A 10-year-old female had a blunt abdominal trauma with macro-hematuria. The computed tomography scan revealed the presence of a horseshoe kidney and a $3^{\text {rd }}$ grade renal lesion and contrast leakage from the right ureter. The ureteral rupture was confirmed by cystoscopy and ascendant pyelography and than a double J-stent was implanted. The stent was removed one month later. Non-surgical management has become the standard of care for both ureteral and renal lesions in children. Non-surgical treatment is a safe procedure for renal trauma with ureteral rupture in children.
\end{abstract}

\section{Introduction}

Blunt abdominal trauma causes renal injury in 10 to $20 \%$ of cases and more than $90 \%$ of renal injuries, in the paediatric population, result from blunt trauma. ${ }^{1}$ Pre-existing congenital or acquired renal and urinary tract abnormalities are more susceptible to injury than normal ones.1-3 We report a case which highlights the association between blunt abdominal trauma, renal and ureteral rupture in a patient with unknown horseshoe kidney.

\section{Case Report}

In September 2013 a 10-year-old female was admitted to our Department after a severe right flank pain due to a blunt abdominal

Correspondence: Nicola Zampieri, Pediatric Surgical Unit, Department of Surgical Sciences, G.B. Rossi Hospital, Piazzale L.A. Scuro 1, 37134 Verona, Italy. Tel: +39.045 .8124916 - Fax: +39.045 .8124662 .

E-mail: dr.zampieri@libero.it

Key words: Blunt trauma; Children; Horseshoe kidney; Ureteral lesion.

Received for publication: 4 March 2015.

Accepted for publication: 16 July 2015.

This work is licensed under a Creative Commons Attribution NonCommercial 3.0 License (CC BY-NC 3.0).

(C) Copyright A. Mariotto et al., 2015

Licensee PAGEPress, Italy

La Pediatria Medica e Chirurgica 2015; 37:110

doi:10.4081/pmc.2015.110 trauma. She was hemodynamically stable, and thoracic and cardiovascular examinations were normal. She had macro-hematuria after the trauma, as well as after catheterization in the emergency room. Blood sample tests were normal, while abdominal ultrasounds (US) revealed the presence of a $40 \times 30 \mathrm{~mm}$ hyperechogenic area reaching the retroperitoneal large vessels. To better classify the parenchymal lesion, we decided to perform an abdominal computed tomography (CT)-scan. The CT-scan defined a $3^{\text {rd }}$ grade renal lesion ${ }^{4}$ and revealed the presence of a horseshoe kidney. A contrast medium leakage from the proximal third of the right ureter was detected, suggesting the ureter rupture (Figure 1).

The day after the admission she underwent a cystoscopy with an ascendant pyelography, which showed contrast leakage. A double Jstent (ch.3) was successfully implanted in the right ureter, under radiologic guide. Two days later an abdominal US was repeated to evaluate the double J-stent position (Figure 2). We performed another CT-scan before discharge. A reduction in the size of the hypodense area (25x17mm) and no more leakage from the right ureter was reported. The right peri-renal spillage was completely absorbed (Figure 3). She was discharged with antibiotic and antifungal therapy one day after and the stent was removed one month after trauma by cystoscopy under anesthetic sedation.

\section{Discussion}

The presence of genitourinary anomalies is likely to increase the risk of genitourinary injury in blunt abdominal trauma. ${ }^{5}$ Cysts and tumors, horseshoe or ectopic kidney, and hydronephrosis are the most common preexisting anomalies detected. Rupture of urinary tract in congenital ureteropelvic junction (UPJ) obstruction is more likely because the hydronephrosis is associated with a high-pressure system, non-compressible, which is prone to rupture after an increase in intra-abdominal pressure due to the blunt trauma.

The location of horseshoe kidneys and the location of the isthmus, in front of the lumbar vertebrae, made them subject to a higher risk of injuries. Ureteral injuries are uncommon; thus, there is paucity of evidence-based data on management and outcomes of these injuries. Recently, a large retrospective analysis ${ }^{6}$ of the American National Trauma Data Bank from 2002 to 2006 has provided additional data on the characteristics and outcomes of 582 patients with ureteral injury. Ureteral injury occurred more commonly in penetrating than in blunt trauma (a ratio of approximately 2:1). The treatment of ureteral ruptures depends on the extent of the lesion and on the amount of urine leakage. Nowadays, according to the hemodynamic stability of the child and the CT findings, the standard of care is the conservative and minimally invasive approach, both in ureteral and in renal lesions. The positioning of a double $\mathrm{J}$-stent is the practice most frequently used to ensure effective and spontaneous repair of the ureteral lesion. 


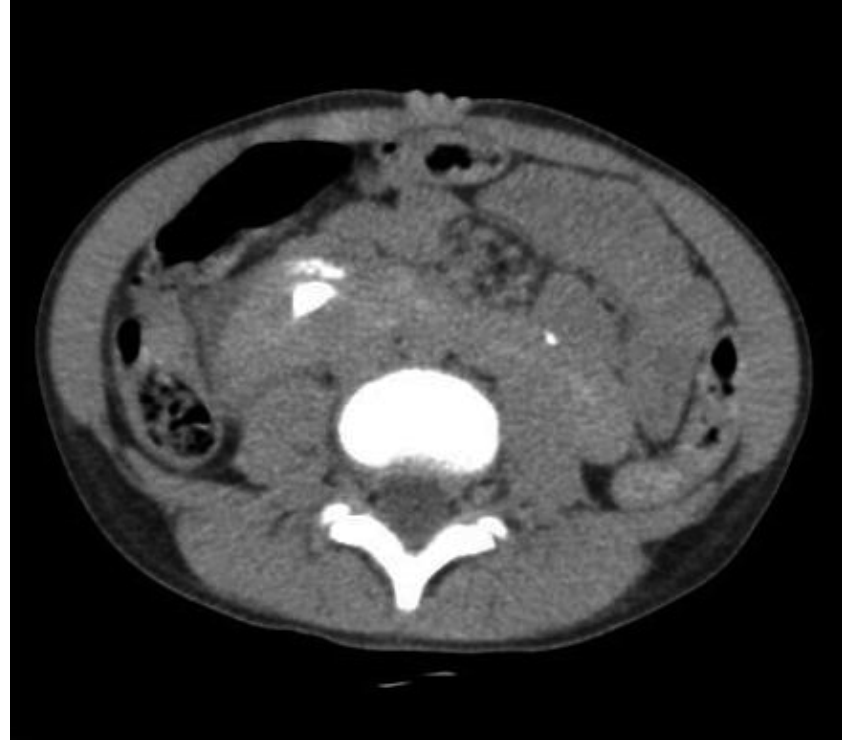

Figure 1. Computed tomography scan showing the ureteral leakage.

Non-operative management has become the standard of care for all solid organ injuries in children. This trend has resulted in renal salvage in $95 \%$ of renal trauma. ${ }^{7}$ Injury to a horseshoe kidney is rare but potentially difficult to manage. Failure of initial conservative management due to deferred bleeding occurs in less than $10 \%$ of cases. Prognostic factors include mainly early detection of the lesion and the precise staging. ${ }^{8}$ Early recognition and treatment of a patient with a previously undiagnosed ruptured horseshoe kidney may represent a serious surgical challenge. ${ }^{2}$ Ultrasonography may provide crucial information on an urinary tract injury, but the abdomino-pelvic CT-scan is the gold standard for studying parenchymal lesions, urinary extravasation and fluid collection, vascular lesions and perirenal hemorrhages and for detecting renal malformations. ${ }^{9}$

\section{Conclusions}

This case demonstrates that non-surgical treatment is effective and safe for renal and ureteral trauma, even in patients with kidneys and urinary tract congenital anomalies.

\section{References}

1. Murawski M, Gołebiewski A, Komasara L, et al. Rupture of the normal renal pelvis after blunt abdominal trauma. J Pediatr Surg 2008;43:e31-3.

2. McAleer IM, Kaplan GW, LoSasso BE. Congenital urinary tract anomalies in pediatric renal trauma patients. J. Urology 2002;168:1808-10.

3. Wessel LM, Scholz S, Jester I, et al. Management of kidney injuries in children with blunt abdominal trauma. J Pediatr Surg 2000;35:1326-30.

4. Tinkoff G, Esposito TJ, Reed J, et al. American association for the

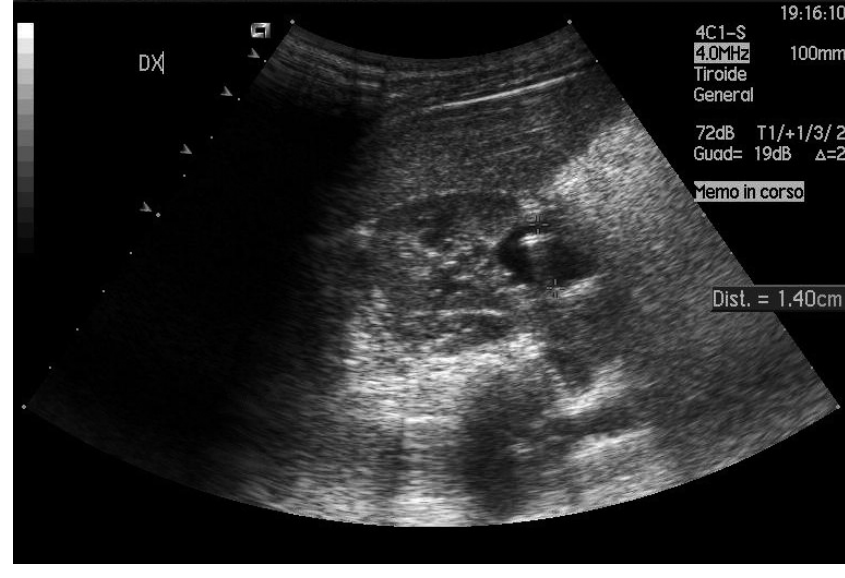

Figure 2. Ultrasound view of stent placement in the renal pelvis.

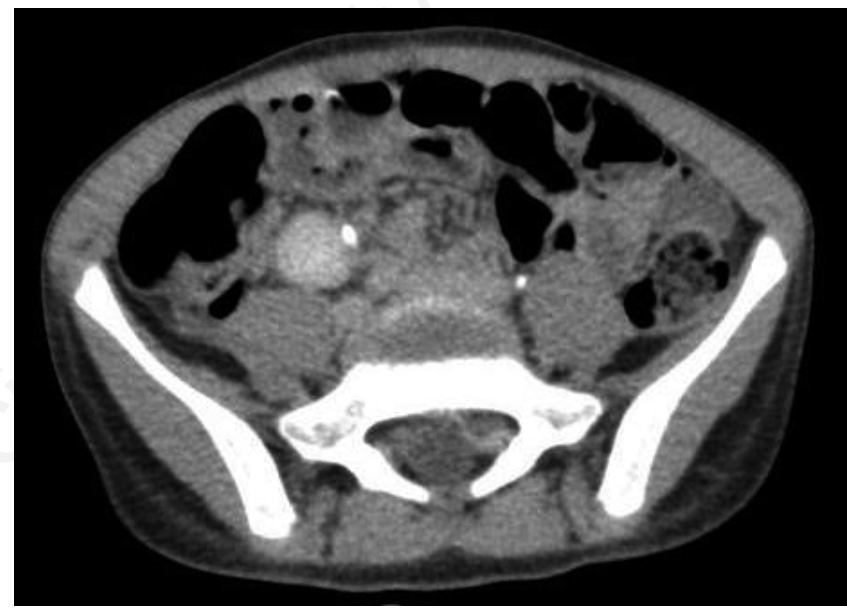

Figure 3. Computed tomography scan showing absence of leakage from the right ureter. surgery of trauma organ injury scale I: spleen, liver, and kidney, validation based on the National Trauma Data Bank. J Am Coll Surgeons 2008;207:646-55.

5. Salem HK, Morsi HAA, Zakaria A. Management of high-grade renal injuries in children after blunt abdominal trauma: experience of 40 cases. J Pediatr Urol 2007;3:223-9.

6. Smith M, Johnston B, Wessells $\mathrm{H}$, et al. Rupture of a ureteropelvic junction. Obstructed kidney in a 15-year-old football player. Am J Roentgenol 2003;180:504.

7. Graziano KD, Juang D, Notrica D, et al. Prospective observational study with an abbreviated protocol in the management of blunt renal injury in children. J Pediatr Surg 2014;49:198-200.

8. Li W-M, Liu C-C, Wu W-J, et al. Rupture of renal pelvis in an adult with congenital ureteropelvic junction obstruction after blunt abdominal trauma. Kaohsiung J Med Sci 2007;23:142-6.

9. Sataa S, Mizouni H, Mohamed H, et al. Blunt trauma causing horseshoe kidney rupture: interest of conservative management. Tunis Med 2013;91:425-7. 